\title{
Periádica Eletrânica

\section{APLICAÇÃO DA TEORIA FUZZY EM MODELOS DE TRANSPORTE DE POLUENTES EM RIOS, PARA ESTUDAR FUNÇÕES DE PERTINÊNCIA RELACIONADAS COM CAMPOS DE CONCENTRAÇÃO, EM FUNÇÃO DAS CARACTERÍSTICAS DO RIO}

\author{
Ticiana Fontoura Vidal ${ }^{1}$ \\ Patrícia Freire Chagas ${ }^{2}$ \\ Raimundo Oliveira de Souza ${ }^{3}$
}

\section{RESUMO}

Neste trabalho, desenvolveu-se uma metodologia que combina a teoria fuzzy com os processos de transporte de poluente, para estudar os campos de concentração em rios, do ponto de vista de incertezas. A metodologia emprega fundamentos da teoria fuzzy para avaliar a solução da equação da difusão advectiva e, assim, transformar um campo de concentração, variável no tempo e espaço, em um campo de funções de pertinências, também variáveis no tempo e no espaço. Os resultados permitiram concluir que a aplicação da teoria fuzzy nos sistemas hidrodinâmicos, pode permitir uma extensão desta metodologia para a determinação de campos de risco em sistemas hídricos.

PALAVRAS-CHAVE: Transporte de massa. Análise de Risco. Equação da Difusão.

\section{APPLICATION OF FUZZY THEORY IN POLLUTION TRANSPORT MODELS IN RIVERS TO STUDY RELEVANCE OF RELATED FUNCTIONS OF CONCENTRATION CAMPS, ACCORDING TO THE RIVER CHARACTERISTICS}

\begin{abstract}
In this work, it was developed a methodology that combines fuzzy theory with pollutant transport processes, to study the concentration fields on rivers, from the standpoint of uncertainty. The methodology employs fundamentals of fuzzy theory to evaluate the solution of the advective diffusion equation and thus become a concentration field, variable in time and space, in a field of membership functions, also variable in time and space. The results showed that the application of fuzzy theory in hydrodynamic systems, may allow an extension of this methodology for the determination of risk fields in water systems.
\end{abstract}

KEYWORDS: Mass Transportation. Risk Analysis. Diffusion Equation.

\footnotetext{
${ }^{1}$ Mestre em Engenharia Civil - Saneamento Ambiental, Universidade Federal do Ceará/Doutoranda em Engenharia Civil - Recursos Hídricos. ticianafvidal@yahoo.com.br

${ }^{2}$ Doutora em Recursos Hídricos, Universidade Federal do Ceará. pfchagas@yahoo.com.br

${ }^{3}$ Doutor em Hidráulica e Saneamento, Universidade Federal do Ceará/Professor Titular Departamento de Engenharia Hidráulica e Ambiental. rsouza@ufc.br
} 


\section{APLICACIÓN DE LA TEORÍA FUZZY EN MODELOS DE TRANSPORTE DE LA CONTAMINACIÓN EN RÍOS PARA ESTUDIAR RELEVANCIA DE FUNCIONES RELATIVAS DE CAMPOS DE CONCENTRACIÓN, SEGÚN LAS CARACTERÍSTICAS DEL RÍO}

\section{RESUMEN}

En este trabajo, hemos desarrollado una metodología que combina la teoría difusa con los procesos de transporte de contaminantes, para estudiar los campos de concentración en los ríos, desde el punto de vista de la incertidumbre. La metodología emplea fundamentos de la teoría difusa para evaluar la solución de la ecuación de difusión advectivo y convertirse así en un campo de concentración, variables en el tiempo y el espacio, en un campo de funciones de pertenencia, también variables en el tiempo y el espacio. Los resultados mostraron que la aplicación de la teoría fuzzy en los sistemas hidrodinámicos, puede permitir una extensión de esta metodología para la determinación de campos de riesgo en los sistemas de agua.

PALABRAS-CHAVE: Transito del massa. Análisis de riesgo. Ecuación de la difusión.

\section{INTRODUÇÃO}

Os modelos matemáticos de qualidade de água são capazes de representar um processo físico qualquer através da formulação de um conjunto de equações matemáticas. Como todo sistema natural, os sistemas hídricos são bastante complexos e envolvem a interação entre diversos ramos da ciência, tais como hidrologia, hidráulica, geomorfologia e transporte de massa. A diversidade de parâmetros que são necessários para descrever o ecossistema aquático, os processos físicos e a variabilidade espacial e temporal aumentam as dificuldades da modelagem. Assim, modelar um processo físico, presente em um sistema hídrico qualquer, não representa uma tarefa simples.

Muitas técnicas têm sido desenvolvidas com o objetivo de se quantificar riscos nos mais diversos problemas hídricos. Dentre as importantes teorias disponíveis no trato deste problema, podem-se destacar a teoria probabilística e a teoria fuzzy. A primeira, extremamente utilizada em todos os campos da ciência, baseia-se nos princípios dos métodos probabilísticos para se quantificar o risco, através da avaliação das incertezas. A segunda, a teoria fuzzy, exige, como fundamentação básica, o desenvolvimento de funções especiais, com propriedades intrínsecas à referida teoria, de modo que o risco possa ser avaliado (Chagas, 2005). 
Entendendo que o estudo das incertezas é etapa fundamental para o gerenciamento dos riscos nas questões de qualidade de água, a presente pesquisa busca aplicar a teoria fuzzy, em modelos advectivo-difusivo, para avaliar o comportamento de concentração de poluentes em rios, em uma estrutura fuzzy. Assim, esta pesquisa visou calcular os campos de concentração de poluente mostrando o grau de pertinência de cada concentração mediante o cálculo das funções de pertinência em casa seção do rio e para cada intervalo de tempo desejado. Os resultados permitem conhecer as regiões com cada grau de pertinência da concentração do poluente.

\section{METODOLOGIA}

Para esta pesquisa está sendo usado o modelo de transporte de poluente, constituído da Equação da Difusão Advectiva, com características fuzzy, onde campos de concentrações na forma fuzzy são obtidas, de modo que funções de pertinências em cada seção sejam obtidas. $O$ campo de concentração é calculado mediante da equação da difusão advectiva definida por (JAMES, 1993):

$$
\frac{\partial C}{\partial t}+u \frac{\partial C}{\partial x}=\frac{1}{A} \frac{\partial}{\partial x}\left(A E \frac{\partial C}{\partial x}\right)-K C+S_{D}
$$

onde,

C é a concentração da substância ao longo do canal, em $\mathrm{kg} / \mathrm{m}^{3}$;

u é a velocidade ao longo do canal, em $\mathrm{m} / \mathrm{s}$;

A é a área da seção transversal do canal, em $\mathrm{m}^{2}$;

E é o coeficiente de dispersão longitudinal;

Ké o coeficiente de decaimento da substância, $\mathrm{em} \mathrm{T}^{-1}$;

$S_{D}$ representa o lançamento distribuído ao longo do canal.

Desenvolvendo a equação da difusão advectiva, tem-se:

$$
\frac{\partial C}{\partial t}+u \frac{\partial C}{\partial x}=\frac{1}{A}\left[A E \frac{\partial^{2} C}{\partial x^{2}}+E \frac{\partial C}{\partial x} \frac{\partial A}{\partial x}+A \frac{\partial C}{\partial x} \frac{\partial E}{\partial x}\right]-K C+S_{D}
$$

)

Rearrumando a equação, tem-se: 


$$
\frac{\partial C}{\partial t}+u \frac{\partial C}{\partial x}-\frac{E}{A} \frac{\partial A}{\partial x} \frac{\partial C}{\partial x}-\frac{\partial E}{\partial x} \frac{\partial C}{\partial x}=E \frac{\partial^{2} C}{\partial x^{2}}-K C+S_{D}
$$

)

Colocando o termo $\frac{\partial C}{\partial x}$ em evidência, resulta em:

$$
\frac{\partial C}{\partial t}+\left[u-\frac{E}{A} \frac{\partial A}{\partial x}-\frac{\partial E}{\partial x}\right] \frac{\partial C}{\partial x}=E \frac{\partial^{2} C}{\partial x^{2}}-K C+S_{D}
$$

)

Fazendo $\psi=\left[u-\frac{E}{A} \frac{\partial A}{\partial x}-\frac{\partial E}{\partial x}\right]$, a equação fica:

$$
\frac{\partial C}{\partial t}+\psi \frac{\partial C}{\partial x}=E \frac{\partial^{2} C}{\partial x^{2}}-K C+S_{D}
$$

A formulação fuzzy que foi desenvolvida para esta pesquisa utiliza a concentração lançada e a capacidade de assimilação do corpo hídrico como números fuzzy, representados por funções de pertinência, com valores definidos no intervalo $[0,1]$.

Uma função de pertinência, como o próprio nome diz, é uma função que representa o nível de pertinência dos parâmetros, em um processo físico bem definido. Desta forma, quanto maior for o grau de pertinência desta variável no contexto, maior será o valor da função. É importante lembrar que a imagem desta função é o intervalo fechado de números reais $[0,1]$.

A característica especial da lógica fuzzy (também referida como lógica nebulosa e em alguns casos por teoria das possibilidades) é a de apresentar uma forma inovadora de manuseio de informações imprecisas, de forma muito distinta da teoria das probabilidades. A lógica fuzzy possui um método interessante de compreensão de expressões verbais, ações cotidianas de funcionamento racional, vagas ou imprecisas.

Esta teoria é baseada nos conjuntos fuzzy e suporta os modos de raciocínio que são aproximados, ao invés de exatos, como estamos naturalmente acostumados. Devido a esta propriedade a teoria fuzzy tem encontrado grandes 


\section{Periódica Eletranica

aplicações nas seguintes áreas: Sistemas Especialistas, Linguagem natural, Controle de Processos, Processos de Tomada de Decisão, Raciocínio Aproximado, Reconhecimento de padrões (SAAVEDRA, 2003).

DOU, WOLDT, BOGARDI E DAHAB (1997) modelaram e analisaram incertezas usando os números fuzzy para simular o transporte de poluentes em escoamentos subterrâneos. Depois de obtida a solução numérica, comparou-se os resultados do modelo numérico fuzzy com a solução analítica.

MPIMPAS, ANAGNOSTOPOULOS E GANOULIS (2001) aplicaram essa teoria na região do Golfo Thermaikos - Grécia, para estudar o comportamento das concentrações de poluentes naquela região costeira. Os autores desenvolveram um modelo bidimensional de escoamento e sobre este escoamento foi desenvolvido um conjunto de modelos, onde algumas concentrações de substâncias, presentes naquele corpo hídrico, foram equacionadas com base na teoria fuzzy. Os autores concluíram que a viabilidade desta metodologia é muito relevante e pode trazer avanços significativos.

O conceito central da teoria fuzzy é a definição das funções de pertinência, que representam numericamente o grau na qual um elemento pertence a um conjunto. No caso da teoria dos conjuntos clássicos, o valor da função de pertinência de cada elemento em um conjunto clássico é 1 para membros (aqueles que certamente pertencem) ou 0 para não membros (aqueles que não pertencem). Já na teoria fuzzy, verifica-se que com o aumento do grau de pertinência de um elemento em um conjunto, o valor da função de pertinência para esse elemento também aumenta dentro do intervalo [0,1] (BOGARDI; DUCKSTEIN, 2002).

Num conjunto fuzzy $\tilde{X}$, a cada elemento $\mathrm{x}$, pertencente ao conjunto, existe uma função de pertinência associada:

$$
\tilde{X}=\left\{\left(x, \mu_{\tilde{X}}(x)\right) / x \in X\right\}
$$

onde $\mu_{\tilde{X}}(x)$ é chamada função de pertinência ou grau de pertinência, $\mathrm{h}$, de $\mathrm{x}$ em $\tilde{X}$. Como o valor máximo da função de pertinência é $h=1$, o conjunto é dito normal ou normalizado. 


\section{Periádica Eletrânica

Essas funções de pertinência podem ser descritas por funções: trapezoidal, exponencial ou triangular. O tipo mais simples de função de pertinência é a triangular, ou seja, um que tenha função de pertinência linear em ambos os lados do pico.

No campo da qualidade de água, Ganoulis (1994) mostra um excelente exemplo de aplicação da teoria fuzzy, onde os dados disponíveis não são suficientes para determinar uma função densidade de probabilidade. Neste caso, onde os dados são escassos, utilizou-se uma função de pertinência triangular para representar as incertezas no parâmetro t9o.

Um número fuzzy triangular pode ser caracterizado por três números reais: dois valores de $x$ onde a função de pertinência chega a zero e um onde ela atinge $o$ valor 1. Nesse caso têm-se funções de pertinência lineares em ambos os lados do pico.

Aplicação desta teoria na equação (1) permite que a concentração da substância poluente possa ser considerada como números fuzzy, de modo que a citada equação se transforme (CHAGAS, 2005).

$$
\frac{\partial \tilde{C}}{\partial t}+\tilde{u} \frac{\partial \tilde{C}}{\partial x}=\frac{1}{\tilde{A}} \frac{\partial}{\partial x}\left(\tilde{E} \tilde{A} \frac{\partial \tilde{C}}{\partial x}\right) \pm \tilde{K} \tilde{C}+\tilde{S}_{D}
$$

onde:

$\tilde{A}$ função de pertinência para a área transversal;

$\tilde{Q}$ função de pertinência para a vazão;

$\tilde{y}$ função de pertinência para a profundidade;

$\tilde{q}$ função de pertinência para o escoamento lateral;

$\tilde{S}_{0}$ função de pertinência para a declividade de fundo;

$\tilde{S}_{f}$ função de pertinência para a declividade da linha d'água;

$\tilde{C}$ função de pertinência para a concentração;

$\tilde{u}$ função de pertinência para o campo de velocidade longitudinal;

$\tilde{E}$ função de pertinência para o coeficiente de dispersão longitudinal;

$\tilde{K}$ função de pertinência para o decaimento;

$\tilde{S}_{D}$ função de pertinência para o lançamento difuso.

Além dessas formulações tem-se ainda, para completar o modelo, o seguinte: Ainda no processo de modelagem com relação aos parâmetros pertinentes 
aos processos de transporte, pode-se definir o coeficiente de dispersão pela relação (FISCHER, 1979):

$$
\tilde{E}=0,05937 \frac{\tilde{Q}}{\tilde{S}_{0} B}
$$

onde B é a largura do rio.

A solução deste conjunto de equações permite determinar as variáveis dependentes, na forma de funções de pertinências. Essas funções são calculadas ao longo de trechos do rio para diferentes tempos. Assim, a equação fuzzy da hidrodinâmica produz um campo de escoamento fuzzy definido por uma função de pertinência para a vazão, uma função de pertinência para a área da seção transversal e uma função de pertinência para a velocidade.

\section{RESULTADOS}

O processo de simulação, através do programa computacional desenvolvido, foi realizado com o objetivo de calcular o campo de concentração em um trecho de um rio sujeito a lançamentos. O modelo foi testado e comparado com resultados da literatura e os resultados foram bastante satisfatórios. Finalmente o modelo foi aplicado para o Rio Pontengi no estado do Rio Grande do Norte, considerando duas condições extremas para este rio. Na primeira simulação foram consideradas as condições de seca ou de estiagem para a região. Na segunda foram consideradas condições chuvosas para o mesmo rio.

Para testar a capacidade do modelo numérico fuzzy, simulou-se um exemplo simples de transporte de poluentes proposto por (DOU et al., 1997). Nesta simulação considerou-se uma condição de contorno para concentração de $100 \mathrm{mg} / \mathrm{L}$ e o comprimento do canal de $1525 \mathrm{~m}$. Na discretização, o canal é dividido em 100 trechos de 15,25 m de comprimento cada, com 1000 intervalos de tempo de 1 dia. Ainda, como dados de entrada foram utilizadas funções fuzzy triangulares para os parâmetros velocidade $(\mathrm{V})$, fator de ponderação para a dispersão $(\alpha)$, Coeficiente de dispersão $\left(\mathrm{E}_{\mathrm{L}}\right)$, a saber, $\mathrm{V}=[1,8 ; 2,4 ; 3,1] \mathrm{m} / \mathrm{s}, \alpha=[9,2 ; 15,3 ; 19,8] \mathrm{m}, \mathrm{E}_{\mathrm{L}}=[16,56$; 
$36,72 ; 61,38] \mathrm{m}^{2} /$ dia. Verifica-se que as funções de pertinência triangulares são usadas para caracterizar os valores das entradas fuzzy nas simulações.

A partir da definição dos números fuzzy triangulares, com seus valores mínimo, médio e máximo, o programa gerou novos valores para as funções de pertinência, com cinco níveis diferentes $0-0.25-0.50-0.75-1$, totalizando 9 valores de concentração. As concentrações foram calculadas a uma distância de $1220 \mathrm{~m}$ da origem.

Tabela 1 - Função de Pertinência de $\mathrm{C} / \mathrm{C}_{0}$ para $\mathrm{t}=400$ e 800 dias.

\begin{tabular}{|l|c|c|c|c|c|c|c|c|c|}
\hline & $\mathbf{0}$ & $\mathbf{0 , 2 5}$ & $\mathbf{0 , 5 0}$ & $\mathbf{0 , 7 5}$ & $\mathbf{1}$ & $\mathbf{0 , 7 5}$ & $\mathbf{0 , 5}$ & $\mathbf{0 , 2 5}$ & $\mathbf{0}$ \\
\hline $\mathbf{T}=\mathbf{4 0 0 d}$ & 0 & 0,001 & 0,006 & 0,026 & 0,076 & 0,174 & 0,313 & 0,477 & 0,649 \\
\hline $\mathbf{T}=\mathbf{8 0 0 d}$ & 0,816 & 0,925 & 0,975 & 0,993 & 0,999 & 1 & 1 & 1 & 1 \\
\hline
\end{tabular}

A Tabela 1 e a Figura 1 mostram os valores das funções de pertinência da concentração no mesmo ponto para os tempos de 400 e 800 dias.

Figura 1 - Funções de pertinência, para as concentrações, obtidas pelo modelo desenvolvido, para os tempos de 400 e 800 dias.

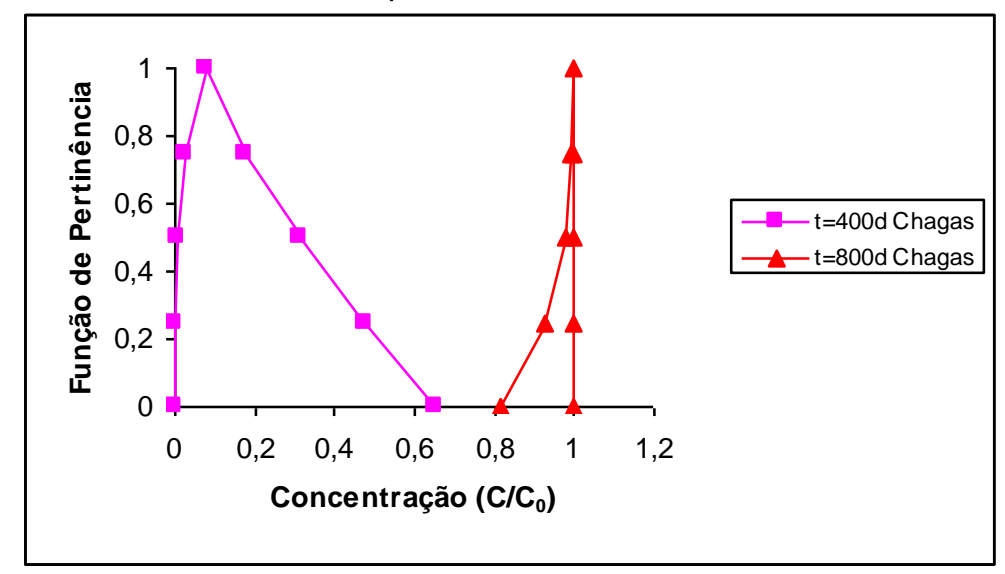

A Figura 1 mostra a comparação entre as funções de pertinência da concentração obtidas através do modelo desenvolvido nesta pesquisa, com as funções obtidas da solução numérica do modelo de Dou et al. (1997). Verifica-se que os valores do modelo desenvolvido coincidem quase que integralmente com os resultados de Dou et al. (1997) mostrando assim a eficiência do modelo. 
Figura 2 - Comparação das funções de pertinência para o modelo desenvolvido e o modelo de DOU et al. (1997), para os tempos de 400 e 800 dias.

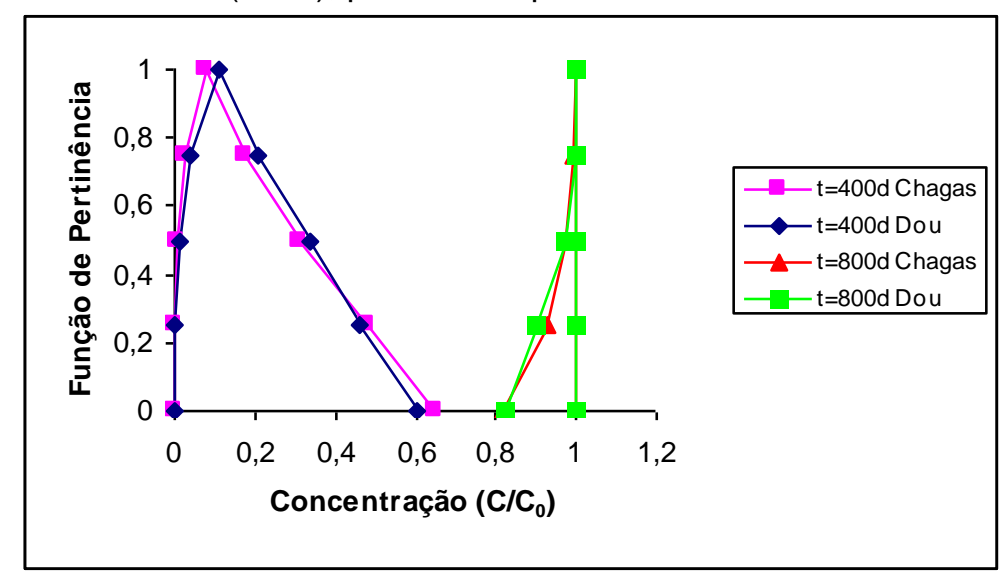

A Figura 2 também apresenta os valores das concentrações calculadas, em cada nível, para diferentes tempos, entre os níveis 0 e 1 . Assim, têm-se os valores: baixo $\left(\mathrm{C}_{\mathrm{L}}\right)$, médio $\left(\mathrm{C}_{\mathrm{M}}\right)$ e alto $\left(\mathrm{C}_{\mathrm{U}}\right)$ da concentração.

Um a vez comprovada a eficiência do modelo, o mesmo foi aplicado no rio Potengi e algumas simulações foram realizadas. Nas simulações, considerou-se 0 lançamento de esgotos de origem doméstica para avaliar o comportamento da concentração ao longo do rio. Dois indicadores de qualidade de água, OD e coliformes fecais, foram utilizados.

Os dados do rio Potengi, utilizados nas simulações seguintes, são: comprimento do canal $9 \mathrm{~km}$, temperatura da água $30^{\circ} \mathrm{C}$ e largura do canal $37 \mathrm{~m}$. As funções de pertinência do coeficiente de rugosidade e declividade do canal são $[0,0075 ; 0,01 ; 0,0125]$ e $[0,00003 ; 0,00004 ; 0,00005] \mathrm{m} / \mathrm{m}$, respectivamente. As simulações foram realizadas para uma situação mais favorável, onde foi considerada uma vazão de $14,5 \mathrm{~m}^{3} / \mathrm{s}$ e, uma situação desfavorável, com uma vazão de $2,6 \mathrm{~m}^{3} / \mathrm{s}$. Não foram considerados os efeitos de marés.

As figuras seguintes mostram simulações onde foram considerados quatro diferentes lançamentos de coliformes no rio Potengi, $C=500 \mathrm{org} / 100 \mathrm{~mL}$, $\mathrm{C}=750 \mathrm{org} / 100 \mathrm{~mL}, \mathrm{C}=1000 \mathrm{org} / 100 \mathrm{~mL}$ e $\mathrm{C}=1500 \mathrm{org} / 100 \mathrm{~mL}$. A função de pertinência usada para a condição inicial de lançamento é $\left[0,75 \mathrm{C}_{0} ; \mathrm{C}_{0} ; 1,25 \mathrm{C}_{0}\right]$ org $/ 100 \mathrm{~mL}$. $A$ função de pertinência de resistência é $[20,200,500]$ org/ $100 \mathrm{~mL}$. 
Figura 3 - Representação fuzzy da concentração final de coliformes no rio Potengi, em $x=4,5 \mathrm{~km} e$ $\mathrm{t}=8 \mathrm{~h}$, com $\mathrm{Q}=2,6 \mathrm{~m}^{3} / \mathrm{s}$ para 4 lançamentos distintos.

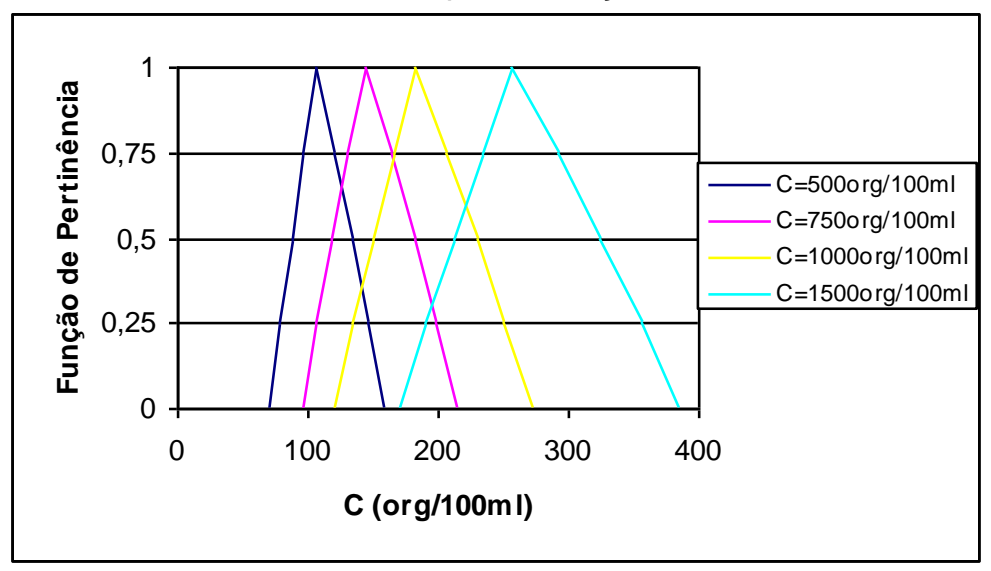

As Figuras 3 e 4 mostram as funções de pertinência calculadas para um tempo de observação de 8 horas após o lançamento, para uma seção a 4,5km da origem do canal, considerando os dois cenários de vazão. Através das figuras podese observar que para o cenário de maior vazão (fig. 4) o grau de maior pertinência para a concentração varia entre 60 e 105 org/100mL, correspondente aos lançamentos de 500 e 1500 org/100mL. Já para o cenário mais desfavorável, vazão de $2,6 \mathrm{~m} / \mathrm{s}$, o grau de maior pertinência varia entre 100 e $280 \mathrm{org} / 100 \mathrm{~mL}$. Verificase, então, que para um mesmo lançamento a capacidade de diluição do rio Potengi torna-se bem menor. Este resultado permite concluir que a função risco tende a aumentar nos períodos de seca.

Figura 4 - Representação fuzzy da concentração final de coliformes no rio Potengi, em $x=4,5 \mathrm{~km}$ e $\mathrm{t}=8 \mathrm{~h}, \mathrm{com} \mathrm{Q}=14,5 \mathrm{~m}^{3} / \mathrm{s}$ para 4 lançamentos distintos.

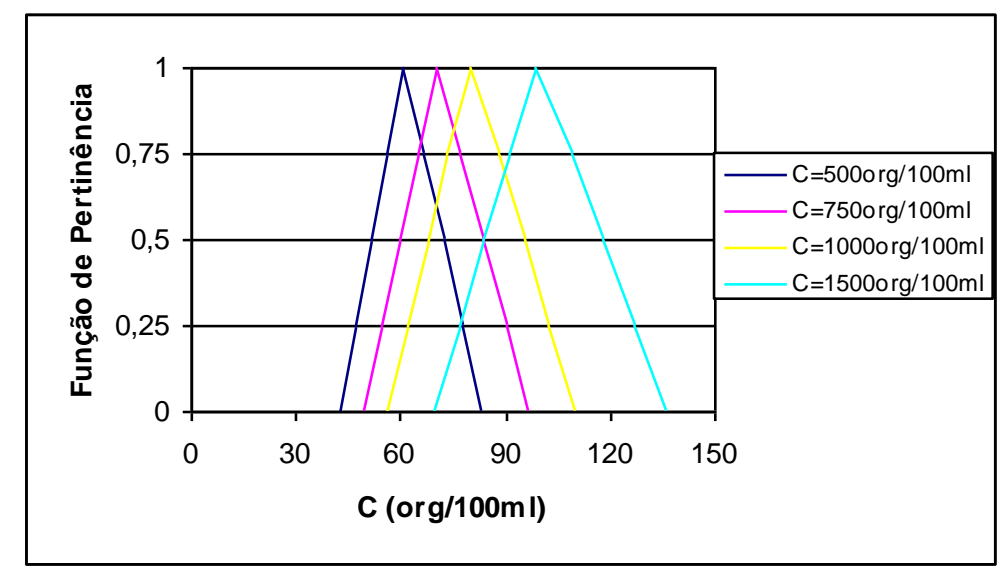


Figura 5 - Representação fuzzy da margem de segurança do sistema, em $x=4,5 \mathrm{~km}$ e t=8h, com $\mathrm{Q}=2,6 \mathrm{~m}^{3} / \mathrm{s}$ para 4 lançamentos distintos.

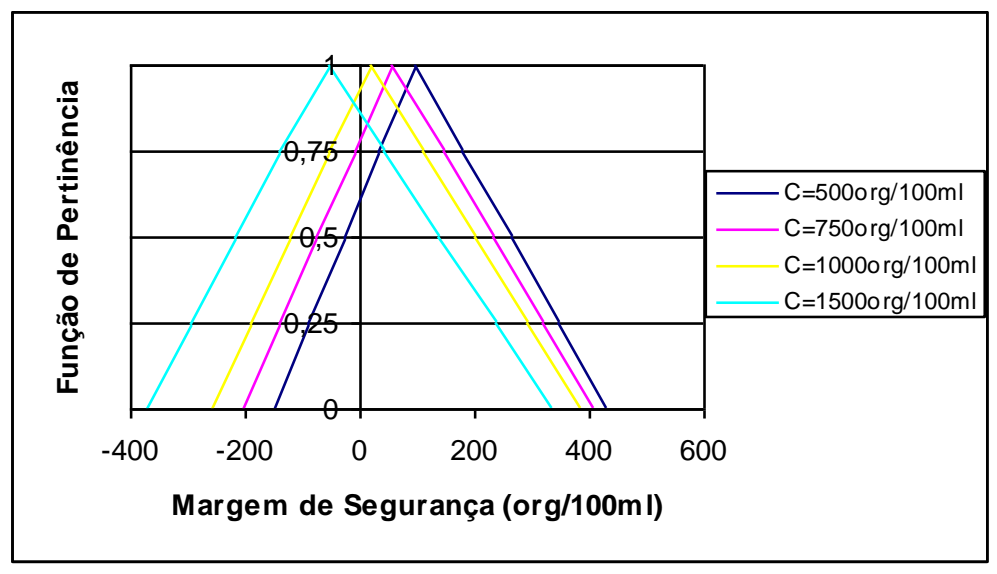

Os resultados das funções de pertinência para a margem de segurança, apresentados nas figuras 5 e 6, mostram que quanto maior o lançamento mais para a esquerda se desloca esta função, caracterizando assim um maior risco de falha para o sistema. Entretanto, a comparação das referidas figuras permite concluir que para maiores vazões este deslocamento é bem menor.

Figura 6 - Representação fuzzy da Margem de segurança do sistema, em $x=4,5 \mathrm{~km}$ e $\mathrm{t}=8 \mathrm{~h}$, com $\mathrm{Q}=14,5 \mathrm{~m}^{3} / \mathrm{s}$ para 4 lançamentos distintos.

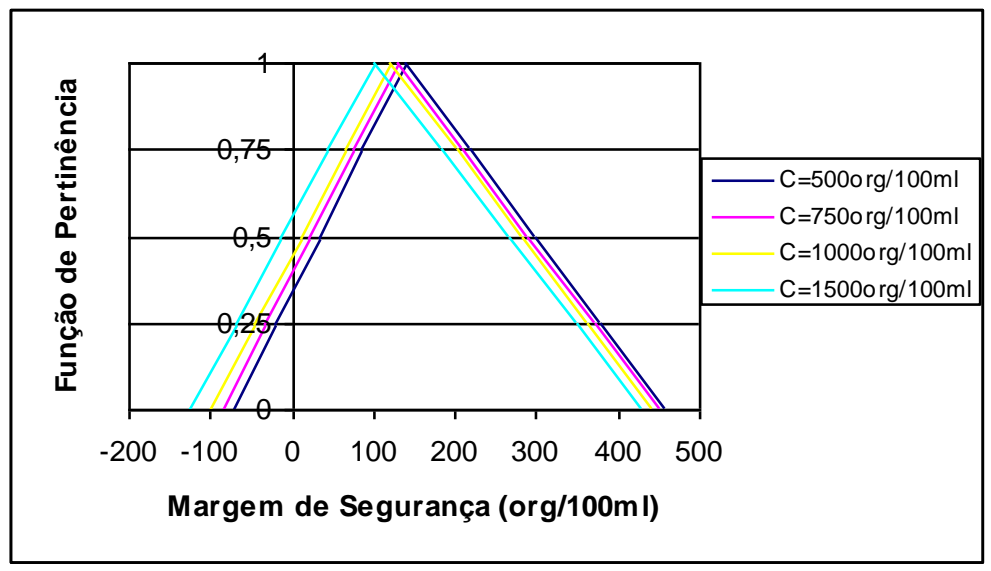

A Figura 7 faz uma comparação entre as concentrações finais do sistema, considerando os dois cenários de vazões: $2,6 \mathrm{~m}^{3} / \mathrm{s}$ e $14,5 \mathrm{~m}^{3} / \mathrm{s}$. Pode-se observar que há um deslocamento para a esquerda da função fuzzy para maiores vazões. 
Figura 7 - Comparação entre as concentrações finais do sistema, para duas vazões 2,6 e $14,5 \mathrm{~m}^{3} / \mathrm{s}$, em $\mathrm{x}=4,5 \mathrm{~km}$ e $\mathrm{t}=8 \mathrm{~h}$, para $\mathrm{C}_{0}=1500$ org $/ 100 \mathrm{~mL}$.

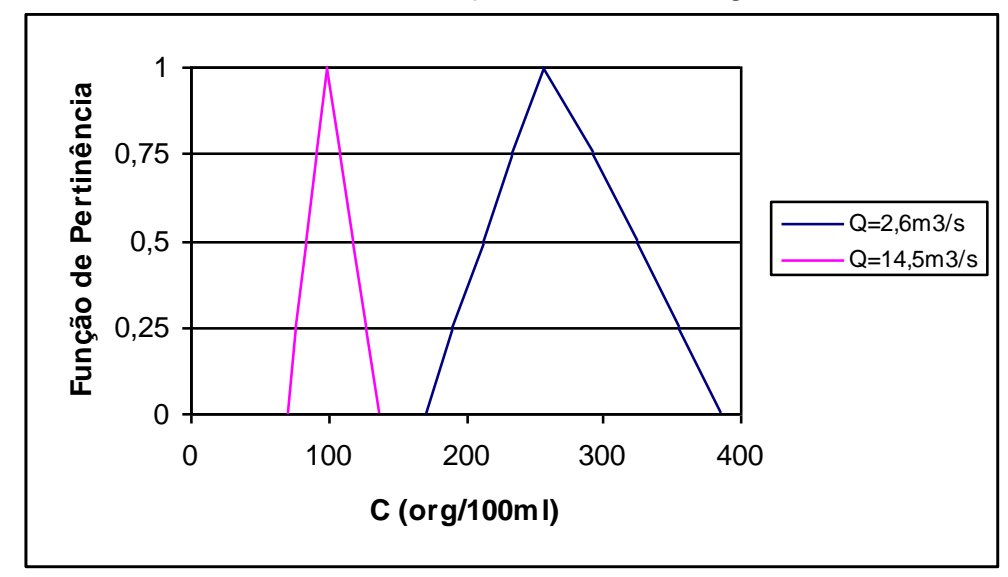

Em contrapartida, os resultados apresentados na Figura 8, mostram que rios com maiores vazões têm uma função marginal de segurança centrada mais para a direita, fato este que afeta significativamente o comportamento da função risco.

Figura 8 - Comparação entre as margens de segurança do sistema, para duas vazões 2,6 e 14,5 $\mathrm{m}^{3} / \mathrm{s}$, em $\mathrm{x}=4,5 \mathrm{~km}$ e $\mathrm{t}=8 \mathrm{~h}$, para $\mathrm{C}_{0}=1500$ org $/ 100 \mathrm{~mL}$.

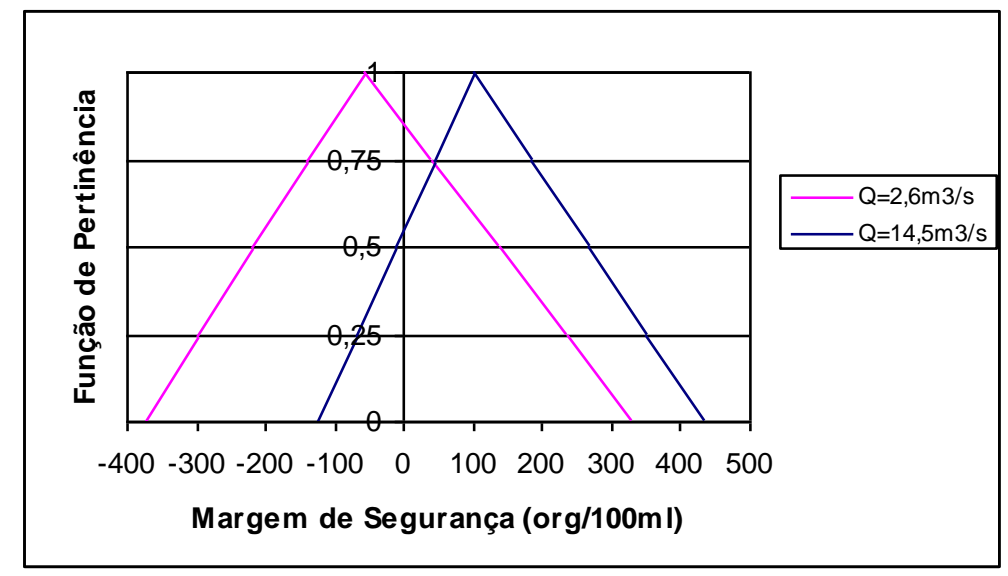

As Figuras 9 e 10 apresentam os resultados obtidos para as funções de pertinência do oxigênio dissolvido para um tempo de 8 horas, em uma seção a $4,5 \mathrm{~km}$ do ponto de lançamento e para os cenários de escoamentos anteriores. Verifica-se que para os vários lançamentos de DBO a variação das funções de pertinência é mais latente no cenário de baixa vazão. Neste caso, quando o lançamento varia de $50 \mathrm{mg} / \mathrm{L}$ para $200 \mathrm{mg} / \mathrm{L}$, a concentração com maior grau de 
pertinência varia de $6 \mathrm{mg} / \mathrm{L}$ para $7 \mathrm{mg} / \mathrm{L}$. Já para o cenário de estação chuvosa, a concentração com maior grau de pertinência fica em torno de $7,2 \mathrm{mg} / \mathrm{L}$.

Figura 9 - Representação fuzzy da concentração final de OD no rio Potengi, em x=4,5km e t=8h, com $\mathrm{Q}=2,6 \mathrm{~m}^{3} / \mathrm{s}$ para 4 lançamentos distintos.

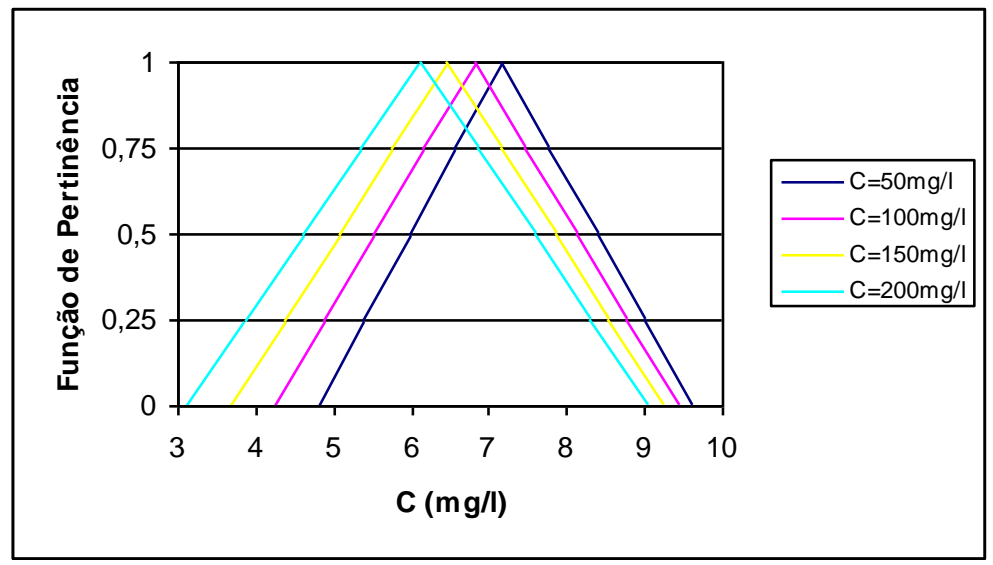

Figura 10 - Representação fuzzy da concentração final de OD no rio Potengi, em x=4,5km e t=8h, com $\mathrm{Q}=14,5 \mathrm{~m}^{3} / \mathrm{s}$ para 4 lançamentos distintos.

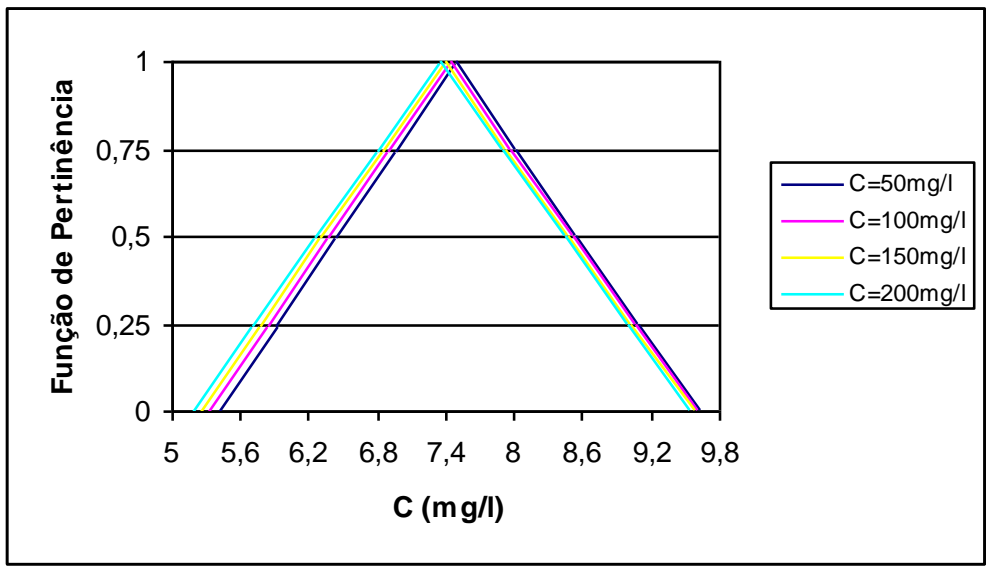

\section{CONCLUSÃO}

As simulações mostraram que esta função de pertinência desempenha importante papel na avaliação do risco. Em consequência, uma definição adequada para a mesma é de fundamental importância para se obter resultados satisfatórios. Os resultados mostraram que para uma função de pertinência em que o valor da variável com maior comprometimento aumenta, o campo de risco diminui em intensidade.

Para um mesmo tipo de lançamento, rios com maiores vazões produziram campos de riscos menores. Enquanto que, para alguns rios estudados, o mesmo lançamento produziu um campo de risco que chegou próximo a seu estado de saturação. Isto permitiu concluir que, nas questões de controle da conservação 
hidroambiental, aspectos hidráulicos e hidrológicos desempenham papéis fundamentais.

Com relação ao rio Potengi, as análises mostraram que a capacidade de diluição do mesmo sofreu uma variação significativa quando houve mudanças bruscas na vazão. Para o período de seca, verificou-se que o rio fica bastante vulnerável a lançamentos com concentrações de coliformes e DBO, com as características dos esgotos domésticos. Neste caso, os resultados mostraram que as concentrações, para pequenas vazões deixam o rio altamente vulnerável. Esta análise permite concluir que para os períodos de seca, há a necessidade de um rigoroso controle nos lançamentos dos esgotos domésticos, de modo a reduzir as chances de contaminação daquele corpo hídrico.

Finalmente, uma análise global da metodologia apresentada permite verificar a grande versatilidade da teoria fuzzy no cálculo de campo de concentrações provenientes de modelos matemáticos fuzzy. A pesquisa mostrou que é possível fazer uma análise de incertezas, com a mesma eficiência de outras técnicas que demandam grandes quantidades de dados.

\section{REFERÊNCIAS}

BOGARDI, I., DUCKSTEIN, L. The Fuzzy Logic paradigm of Risk Analysis. In: Risk-Based Decisionmaking In Water Resources X. Santa Barbara, California, 2002. pp. 12-22.

CHAGAS, P. F. Perspectivas da Aplicação da Teoria Fuzzy para o cálculo de risco em sistemas hidrodinâmicos. Tese defendida no Departamento de Engenharia Hidráulica e Ambiental da Universidade Federal do Ceará como parte dos requisitos para obtenção do título de doutor em recursos hídricos, 2005.

FISCHER, H. B. Mixing in Inland and Coastal Water, Academic Press, Inc, 1979.

GANOULIS, J.; DUCKSTEIN, L.; BOGARDI, I. Risk Analysis of Water Quantity and Quality Problems: The Engineering Approach. In: Ganoulis (Ed.), Water Resources Engineering Risk Assessment, Nato ASI Series, Serie G: Ecological Sciences, v. 29, 1991.

GANOULIS, J. G. Engineering Risk Analysis of Water Pollution: Probabilities and Fuzzy sets. VCH publishers Inc. - Weinheim; New York; Basel; Cambridge; Tokyo: 1994.

JAMES, A. An Introduction to Water Quality Modelling. 2nd Edition. By John Wiley \& Sons Ltd, 1993. 311p.

MPIMPAS, H.; ANAGNOSTOPOULOS, P.; GANOULIS, J. Modelling of water pollution in the Themaikos Gulf with fuzzy parameters. Ecological Modelling, n. 142, 2001. pp. 91-104. 
Volume 11, Número 06, 2015

Planejamento e Gestão dos Recursos Hídricos

SAAVEDRA, O. R. Introdução aos Conjuntos Difusos - Notas de aula - Inteligência Artificial. Planejamento e Gestão dos Recursos Hídricos. Universidade Federal do Maranhão, 2003. 